\section{Absorption Spectra observed during the Flash Photolysis of Mixtures of Carbonyl Sulphide and Nitrogen Trifluoride}

THE isothermal flash photolysis of mixtures of nitrogen trifluoride and carbonyl sulphide, and nitrogen trifluoride and carbon disulphide, has been investigated, as part of a study of the reactions of atomic sulphur with a series of volatile inorganic fluorides. The ground state $\left({ }^{3} P\right)$ sulphur atom, obtained on photolysing carbon disulphide with light of wavelength $<2200 \AA$, was found to be unreactive towards nitrogen trifluoride, while the excited $\left({ }^{1} D\right)$ atom, obtained on photolysing carbonyl sulphide, was found to react slowly.

Nitrogen trifluoride prepared by the electrolysis of molten ammonium hydrogen fluoride, carbonyl sulphide obtained from the reaction of concentrated sulphuric acid with a cold concentrated solution of potassium thiocyanate and carbon disulphide were purified by distillation in vacuo until their high pressure infra-red spectra showed the absence of impurities. The flash photolysis apparatus used was identical to that described elsewhore ${ }^{1}$ and spectra were recorded on Ilford $Q$ and Ilford 'Selochrome' plates using a Littrow type spectrograph (Hilger $E .742$ ).

Absorption spectra obtained at short delay times after a mixture of carbonyl sulphide, nitrogen trifluoride and argon (in the ratio $1: 2: 20$ ) had been flash photolysedusing $5 \mathrm{~mm}$ mercury partial pressure of carbonyl sulphide -showed strong $\mathbf{S}_{2}$ absorption which was observed to reach its maximum intensity at the shortest delay time used, that is, $15 \mu \mathrm{sec}$. In addition, a new complex series of weak bands was obtained in the region $3300-4200 \AA$. As a result of the weak nature of these bands, it was difficult to obtain accurate measurements of the wavelengths. Attempts to improve the intensity of the system by varying the ratio of the mixture were unsuccessful. Table 1 lists the band head wavelengths and intensities (scale of ten) - all bands were degraded to the red.

It was not possible to fit all the bands into one vibrational scheme; however, they could be arranged in two systems, that is, $3286 \cdot 5-3655 \cdot 7 \AA$ and $3703 \cdot 1-4189 \cdot 4 \AA$. The mean differences in the $3286.5 \AA$ system are $440 \mathrm{~cm}^{-1}$ and $644 \mathrm{~cm}^{-1}$, while those of the $3703 \cdot 1 \AA$ system are $412 \mathrm{~cm}^{-1}$ and $634 \mathrm{~cm}^{-1}$. Photometric measurements on the strongest bands of the $3703 \cdot 1 \AA$ system showed that it reached its maximum intensity at about $60-70 \mu \mathrm{sec}$ delay, while the $3286.5 \AA$ system seemed to reach its maximum intensity at about 50-60 $\mu$ sec. It was not possible, however, to carry out accurate photometry on the latter system because of its weak nature. Both systems decayed to zero intensity after $500 \mu$ sec.

Investigation of the spectral region $2200-2800 \AA$ when a mixture of carbonyl sulphide, nitrogen trifluoride and argon (in the ratio $1: 2: 25$ ) was flash photolysed-using $2 \mathrm{~mm}$ mercury partial pressure of carbonyl sulphideshowed in addition to $S_{2}$ the presence of an appreciable concentration of NS in the reaction mixture even at $15 \mu$ sec delay. The NS reached maximum intensity at about 50 usec and had disappeared by $250 \mu$ sec.

A range of mixtures each containing $5 \mathrm{~mm}$ morcury partial pressure of carbonyl sulphide but varying amounts

Table 1. Wavelengths of Band Heads observed during the Flash Photolysis of MtXtures of Carbonyl SUlPhide and Nitrogen Tri-

$\begin{array}{cccc}\begin{array}{c}\text { Wavelength } \\ \text { (in air) } \pm 0 \cdot 2 \AA\end{array} & \begin{array}{c}\text { Relative intensity } \\ \text { (visual cstimate) }\end{array} & \begin{array}{c}\text { Wavelength } \\ \text { (in air } \pm 0 \cdot 2 \AA \text { ) }\end{array} & \begin{array}{c}\text { Relative intensity } \\ \text { (visual estimate) }\end{array} \\ 3286 \cdot 5 & 8 & 3703 \cdot 1 & 7 \\ 3335 \cdot 7 & 8 & 3761 \cdot 6 & 7 \\ 3358 \cdot 6 & 5 & 3818 \cdot 5 & 3 \\ 3384 \cdot 8 & 8 & 3854 \cdot 7 & 7 \\ 3410 \cdot 0 & 5 & 3878 \cdot 4 & 5 \\ 3433 \cdot 7 & 8 & 3916 \cdot 5 & 10 \\ 3460 \cdot 6 & 8 & 3942 \cdot 8 & 5 \\ 3485 \cdot 7 & 8 & 3950 \cdot 2 & 3 \\ 3515 \cdot 6 & 7 & 3979 \cdot 5 & 10 \\ 3540 \cdot 4 & 8 & 4008 \cdot 0 & 10 \\ 3570 \cdot 3 & 6 & 4042 \cdot 0 & 3 \\ 3596 \cdot 4 & 8 & 4083 \cdot 0 & 5 \\ 3655 \cdot 7 & 8 & 4145 \cdot 3 & 3 \\ & & 4189 \cdot 4 & 4\end{array}$

of nitrogen trifluoride (between 0 and $15 \mathrm{~mm}$ mercury) were flash photolysed isothermally, and spectra recorded at $80 \mu \mathrm{sec}$ delay. Although the complex system of bands in the region $3300-4200 \AA$ were too weak for accurate photometry to be carried out, a visual examination of the strongest bands at $3979.5 \AA$ and $4008.0 \AA$ showed that their intensity at $80 \mu$ sec was increased by increasing the partial pressure of nitrogen trifluoride. The intensity of $\mathrm{S}_{2}$, however, was only decreased very slightly by incroasing the partial pressure of nitrogen trifluoride.

All spectra obtained during the experiments were closely examined for the presence of $\mathrm{NF}_{2}$ absorption ${ }^{2}$ at $2600 \AA$, but no banded absorption other than $S_{2}$ was observed in this region at any delay time.

An increase in the partial pressure of nitrogen trifluoride only resulted in slight suppression of the $S_{2}$ intensity, and because of this the reaction of $\mathrm{S}\left({ }^{1} D\right)$ with nitrogen trifluoride must bo slow compared with the reaction of $\mathrm{S}\left({ }^{1} D\right)$ with carbonyl sulphide. The initial reaction of $\mathrm{S}\left({ }^{1} D\right)$ with nitrogen trifluoride could result either in abstraction to give $\mathrm{SF}$ and $\mathrm{NF}_{2}$ radicals or addition to give a "hot" $\mathrm{SNF}_{3}$ * molecule which could then lose energy by decomposition to species such as $\mathrm{SNF}_{2}$ and SNF. The absence of $\mathrm{NF}_{2}$ absorption must result cither from the slow nature of the abstraction reaction or from some fast reaction of $\mathrm{NF}_{2}$ with carbonyl sulphide, $\mathrm{S}_{2}$ or $\mathrm{S}\left({ }^{1} D\right)$.

The new transient $3300-4200 \AA$ system could therefore either arise from unstable fluorides of nitrogen and sulphur (for example, $\mathrm{NF}_{2}, \mathrm{NF}, \mathrm{SF}, \mathrm{SF}_{2}, \mathrm{SF}_{3}$ ) or from species such as $\mathrm{SNF}_{3}, \mathrm{SNF}_{2}$ and SNF. The species $\mathrm{SNF}_{3}$ and SNF have been reported to be relatively stable molecules although their ultra-violet spectra have not been reported in the literature. The ultra-violet spectrum of the $\mathrm{NF}_{2}$ radical has been reported ${ }^{2}$. From the nature of the $3300-4200 \AA$ \& bands it would seem likely that they belong either to two different transitions of a diatomic radical, or to a triatomic radical. They might therefore arise from one of the species $\mathrm{SF}, \mathrm{SF}_{2}$ or $\mathrm{NF}$.

\section{W. D. McGrath \\ T. Morrow}

Department of Chemistry,

The Queen's University of Belfast,

Belfast.

${ }^{1}$ MeGrath, W. D., and Morrow, T., Trans. Farad. Soc. (in the press).

${ }^{2}$ Johnson, F. A., and Colburn, C. 1., J. Amer. Chem. Soc., 83, 3043 (1961).

\section{Vibrational Spectrum of Liquid Crystalline Methyl Stearate}

Methyl Stearate, $\mathrm{CH}_{3}\left(\mathrm{CH}_{2}\right)_{10} \mathrm{COOCH}_{3}$, occurs in the form of liquid crystals between $22^{\circ}$ and $38^{\circ} \mathrm{C}$ (ref. 1). Optical examination of the textures exhibited by the mesophase indicated that the structure is smectic. The infra-red absorption spectra of this compound were reported by Jones et al. ${ }^{2,3}$ for thin films and also in carbon disulphide solution. We have investigated the spectrum of methyl stearate in the liquid crystalline state to ascertain the distinctive characteristics of the spectrum of this phase.

Fig. 1 shows the infra-red spectra of the liquid $(a)$, liquid erystalline $(b)$ and crystalline $(c)$ phases recorded with a 'Zeiss $U R-10$ ' spectrophotometer. The important features of the spectrum of the liquid crystalline phase are summarized here. It was found that these features become increasingly pronounced the lower the temperature of the liquid crystal.

(1) Sharp peaks appear at 764,785 and $813 \mathrm{~cm}^{-1}$ in the spectrum of the liquid crystal. These peaks are absent from the spectrum of the liquid, but present in that of the solid with greater intensity and sharpness.

(2) The peak at about $888 \mathrm{~cm}^{-1}$ is broad in the liquid, but becomes progressively sharper on passing from the liquid through the liquid erystalline to the solid phase. 